\title{
La formulación de preguntas como estrategia didáctica para motivar la reflexión en el aula
}

\author{
The formulation of questions as a didactic strategy to motivate \\ reflection in the classroom
}

\section{A formulação de perguntas como estratégia didática para motivar a reflexão em sala de aula}

\author{
ISSN 1688-9304 - DOI: 10.18861/cied.2020.11.2.2994 \\ Claudine Glenda Benoit Ríos*1 \\ https://orcid.org/0000-0002-1791-2212
}

Fecha de recibido: 17/12/2019

Fecha de aceptado: 04/05/2020

\begin{abstract}
Resumen
Las diversas estrategias que los docentes implementan en el aula tienen como propósito fundamental el aprendizaje significativo de sus estudiantes. Una de ellas es la formulación de preguntas, una estrategia pedagógica que permite no solamente el aprendizaje de contenidos sino la reflexión en el aula. Dicha reflexión surge en los primeros años de formación docente. En este contexto, el presente artículo da cuenta de un estudio empírico en el que participaron 40 estudiantes de pedagogía en lenguaje de una Universidad de la Región del Biobío, Chile. El objetivo general fue analizar la importancia de la pregunta como estrategia didáctica y de reflexión para profesores en formación, a través de un cuestionario de preguntas abiertas y cerradas. La metodología empleada consideró tanto un análisis cualitativo como cuantitativo, bajo un enfoque descriptivo. Los resultados muestran que las preguntas potencian en los profesores en formación las habilidades de argumentar y de explicar, habilidades esenciales en las prácticas docentes. A su vez, la pregunta se manifiesta como una estrategia basada en una relación bidireccional, asociada directamente con la reflexión sistemática y con la adquisición de nuevos aprendizajes significativos para la formación académica. El empleo de esta estrategia en el aula potencia una actitud crítica y fomenta el autoaprendizaje del alumnado, aspectos fundamentales para el asentamiento de saberes permanentes en el tiempo.
\end{abstract}

Palabras clave: pregunta, estrategia didáctica, metacognición, reflexión, aprendizaje significativo.

\begin{abstract}
The diverse strategies that teachers implement in the classroom have as fundamental purpose the meaningful learning of their students. One of them is the formulation of questions, a pedagogical strategy that allows not only the learning of contents, but also the reflection in the classroom. This reflection arises from the first years of teacher training. In this context, this article presents an empirical study in which 40 preservice teachers from a University of the Biobío Region, Chile, participated. The general objective was to analyze the importance of the question as a didactic and reflection strategy for teachers in language training, through a questionnaire of open and closed questions. The methodology used considered both a qualitative and quantitative analysis, with a descriptive approach. The results show that the questions empower teachers in training
\end{abstract}


the skills of arguing and explaining, essential skills in teaching practices. In turn, the question manifests itself as a strategy based on a bidirectional relationship, directly associated with systematic reflection and the acquisition of new meaningful learning for academic training. The use of this strategy in the classroom strengthens a critical attitude and encourages self-learning of students, fundamental aspects for the establishment of permanent knowledge over time.

Keywords: question, didactic strategy, metacognition, reflection, meaningful learning.

\section{Resumo}

As várias estratégias que os professores implementam em sala de aula têm como objetivo fundamental a aprendizagem significativa de seus alunos. Uma delas é a formulação de perguntas, uma estratégia pedagógica que permite não apenas a aprendizagem de conteúdo, mas a reflexão em sala de aula. Essa reflexão surge nos primeiros anos de formação de professores. Nesse contexto, este artigo relata um estudo empírico no qual participaram 40 estudantes de pedagogia na linguagem de uma universidade da região do Biobío, no Chile. O objetivo geral foi analisar a importância da questão como estratégia didática e de reflexão para os professores em formação, por meio de um questionário de perguntas abertas e fechadas. A metodologia utilizada considerou uma análise qualitativa e quantitativa, sob uma abordagem descritiva. Os resultados mostram que as questões aprimoram na formação de professores as habilidades de argumentar e explicar, habilidades essenciais nas práticas de ensino. Por sua vez, a questão se manifesta como uma estratégia baseada em uma relação bidirecional, diretamente associada à reflexão sistemática e à aquisição de novos aprendizados significativos para a formação acadêmica. O uso dessa estratégia em sala de aula aprimora uma atitude crítica e promove a autoaprendizagem do aluno, aspectos fundamentais para o estabelecimento de conhecimento permanente ao longo do tempo.

Palavras-chave: questão, estratégia didática, metacognição, reflexão, aprendizagem significativa.

\section{Introducción}

La dinámica de cuestionar y responder forma parte de todo intercambio comunicativo. Las relaciones que se establecen durante la comunicación son posibles gracias a la formulación de preguntas que nacen de la inquietud por el conocimiento, por descubrir el sentir del otro, por saber qué piensa frente a un tema, qué sabe, qué lo inquieta y se retroalimenta a partir de las respuestas que posibilitan un proceso cíclico, variable y reflexivo. El valor del cuestionamiento está precisamente en su capacidad para la construcción de sentidos, tanto desde un plano personal como social. Para Eshach, Dor-Ziderman \& Yefroimsky (2014) las preguntas son un componente inseparable de la vida humana en tanto contribuyen a un eficiente desarrollo personal y al progreso de la humanidad (p. 67). Como elemento inherente a la vida, está presente en la cotidianeidad y desde ahí se difumina hacia otras áreas de desarrollo particular, como es el ámbito educativo.

En el contexto de este artículo se concibe la pregunta como un enunciado de carácter interrogativo empleado para obtener, por parte del estudiante, información verbal sobre un aspecto de interés para el proceso de enseñanza-aprendizaje. A lo largo del itinerario formativo la utilización de la pregunta se consolida como una estrategia transversal a las diversas disciplinas y áreas de conocimiento y como una actividad permanente del docente en cada una de sus clases. Ellas permiten construir el conocimiento, ampliar los saberes y retroalimentarlos en las distintas fases de aprendizaje. Asimismo, dada su flexibilidad como estrategia pedagógica fomenta el autoaprendizaje (Maarfia, 2017b). 
Cuando los cuestionamientos son pertinentes, tanto por parte del profesor como del estudiante, se instala un recurso didáctico y pedagógico para estimular el proceso de enseñanza-aprendizaje.

Desde una perspectiva de construcción de saberes, el desarrollo de competencias en el estudiantado va ligado a la reflexión constante, a la resolución de problemas ante cada nuevo desafío educativo y al fortalecimiento de las habilidades cognitivo-lingüísticas (García \& Martínez, 2014). Si tales elementos se conjugan a lo largo del proceso formativo es probable que se asiente el aprendizaje significativo. Este término, de gran importancia para entender los diferentes procesos pedagógicos, remite a Ausubel (2009) y conecta la construcción de los conocimientos con las experiencias de valor para el alumnado. Según Caira, Urdaneta \& Mata (2014) este tipo de aprendizaje se lleva a cabo cuando el estudiante "como constructor de su propio conocimiento, establece relaciones entre los conceptos a aprender y les da sentido a partir de la estructura conceptual que ya posee, vale decir, de sus conocimientos previos y de las experiencias que ya tiene" ( $p$. 95). Así, la búsqueda de respuestas a problemáticas asociadas con el trabajo académico y la necesidad de responder a diversos cuestionamientos conforme a su conocimiento y experiencia brindan, entonces, la oportunidad de afianzar un aprendizaje significativo que permita al estudiante desempeñarse eficazmente en distintos contextos.

Si bien uno de los objetivos relacionados con el empleo de la pregunta es el asentamiento del aprendizaje significativo en el alumnado, no todas las interrogantes que se formulan en el aula van dirigidas a la adquisición del mismo. Como estrategia pedagógica la pregunta hace posible reflejar diferentes grados de conocimiento y, al mismo tiempo, desarrollar una o más habilidades cognitivo-lingüísticas. Es un hecho que en años precedentes el foco de la atención estaba en la memorización y repetición de la información. Sin embargo, en la actualidad el interés se centra en el fomento de habilidades superiores de pensamiento $y$, por consiguiente, en el desarrollo del pensamiento crítico (Joseph, 2018). En cada una de las tareas se busca propiciar en el estudiantado el uso de diversas habilidades cognitivo-lingüísticas (Jorba, 2000), en especial de la argumentación puesto que esta es una habilidad de orden superior que se relaciona directamente con el logro de los aprendizajes desde una perspectiva metacognitiva. Su implementación en el aula permite que los estudiantes vayan más allá de la recepción de contenidos de manera pasiva y se configuren como agentes críticos de sus propios procesos.

En ocasiones las respuestas de los estudiantes a interrogantes resultan ser reproductivas, sobre todo frente a aquellas preguntas asentadas en contenidos de carácter conceptual. En el plano del lenguaje los estudiantes se ven motivados a extraer información literal de los textos para entregar un discurso coherente con lo solicitado. En otras situaciones se sienten impulsados a elaborar sus respuestas ciñéndose de manera estricta a los contenidos ya entregados por el profesor. Por ello se vuelve necesario reconocer las implicancias de utilizar cuestionamientos efectivos para la mejora de los aprendizajes ya que la pregunta es concebida "como uno de los medios más eficaces para favorecer el proceso de enseñanza-aprendizaje" (Morón, 2015, p. 1), fundamentalmente de los futuros profesores quienes, al utilizar la estrategia en el aula, podrían fomentar el diálogo permanente y significativo.

Desde sus primeros años de educación formal el niño se enfrenta a la tarea de descifrar los sentidos fundamentales de lo que comienza a aprender. Más tarde, con el aprendizaje de la lectura y de la escritura se fortalecen aquellos aspectos que antes habían sido trabajados de manera oral en el aula y reforzados en casa. En las distintas 
etapas la pregunta es inherente al cumplimiento de los objetivos trazados tanto por el profesor como por la familia. En el plano de la comprensión, "la pregunta interviene en los momentos iniciales del aprendizaje, cuando la actividad de comprender un texto se realiza en forma compartida entre el docente y el alumno" (Arnoux, Nogueira \& Silvestri, 2007, p. 82) y también cuando el estudiante ya es autónomo en la tarea. En el ámbito de la escritura, por su parte, la pregunta es esencial para reforzar la producción textual en forma de cuestionamientos dirigidos al docente, que estimulan la retroalimentación.

Cuando el estudiante ingresa a la universidad afronta el cometido de resolver cuestiones que atañen a su vida académica y que lo obligan a tomar decisiones relevantes para su futuro profesional. Estas decisiones responden a las interrogantes planteadas, las que están mediadas por la ayuda del profesor en tanto facilitador de los aprendizajes. La estrategia en sí es abordada desde la práctica y la experiencia personal, pero luego se amplía a aquellos campos que involucran la realización de predicciones, inferencias y completación de sentidos. Por tal motivo las preguntas en la universidad deben ser contextualizadas y basadas en los conocimientos previos de los educandos. La formulación de interrogantes, por consiguiente, necesita ocurrir en un tiempo y momento definidos para propósitos específicos.

En tal entorno de construcción de conocimientos las creencias que tienen los profesores acerca del proceso de enseñanza-aprendizaje desempeñan un papel crucial en la forma de concebir la enseñanza. Asensio-Muñoz \& Ruiz de Miguel (2017) postulan que "las creencias de los profesores se generan sobre la base de su propio aprendizaje, es decir, las primeras percepciones sobre la enseñanza surgen cuando el profesor es todavía un estudiante" (p. 80). Este proceso de aprendizaje implica la activación de diversos factores en el alumnado, entre ellos los motivacionales, textuales, cognitivos, lingüísticos y metacognitivos. Cada uno determina la forma en que tal estudiante aprende e interactúa con otros en el mismo ambiente de desarrollo de habilidades. En el contexto de la presente investigación se sugiere que el empleo de las preguntas es una estrategia efectiva en el aula puesto que actúa como un factor que potencia el aprendizaje significativo el que, a su vez, estaría caracterizado por la reflexión permanente y sistemática.

\section{Marco teórico. Las preguntas como un elemento potenciador del aprendizaje significativo}

Uno de los desafíos a los que se enfrenta constantemente un docente, aparte de abordar los contenidos de un programa, es identificar las estrategias más efectivas para cumplir los objetivos de aprendizaje en sus estudiantes. Luego de identificarlas debe crear situaciones de aprendizaje para ponerlas en práctica $y$, finalmente, evaluar la efectividad de las mismas para extrapolarlas a nuevos procesos de enseñanza-aprendizaje. La estrategia que media en cada una de las actividades curriculares es la formulación de preguntas, recurso que se instala en la relación entre profesor y alumno, y entre estudiantes con sus pares de colaboración.

Para comprender la forma en que la pregunta puede potenciar el aprendizaje significativo en el aula es indispensable delimitar los alcances de tal concepto. De acuerdo con los planteamientos de Ausubel (2009) el aprendizaje significativo hace referencia a la creación de significados que permiten al estudiante dar valor a su entorno y a las experiencias vividas por él en el aula. En este contexto, cada acercamiento hacia nuevos aprendizajes implicaría una relación dinámica e interactiva entre su conocimiento y la construcción de nuevas realidades. En la búsqueda de estos aprendizajes significativos y duraderos el papel de la pregunta es decisivo dado que se asocia con el descubrimiento y con la búsqueda de razones por las cuales ocurren los fenómenos. Para López, Veit \& Solano 
(2014), "el favorecimiento de un aprendizaje significativo crítico en el aula de clase implica una estimulación del cuestionamiento por parte del estudiante" (p. 118) lo que, a su vez, fortalece su desarrollo cognitivo y social.

Tanto en un aprendizaje tradicional, en el que el profesor tiene un papel protagónico, como en aquel que emana de la reflexión y el autoaprendizaje es fundamental considerar las diferencias individuales entre los estudiantes y, especialmente, tener en cuenta que el estudiantado logra un aprendizaje significativo cuando siente que su participación es valorada por los otros agentes del aula. La pregunta, en este sentido, hace posible un aprendizaje significativo y de provecho para el estudiante. Según López Aymes (2012), "Los alumnos pueden aprender mejor en un ambiente de clase donde sus contribuciones sean valoradas. Tanto el tipo de preguntas que se realicen, como la manera en que se formulen las preguntas y las respuestas dadas afectan la autoestima y la participación del estudiante" (p. 48). La estrategia aquí descrita tiene la versatilidad de acomodarse a diferentes formas de conocimiento y a los diversos modos en que el estudiante participa de su educación formal.

En su formación como futuro profesor el estudiante debería ser capaz de identificar el valor que adquiere el apropiarse de estrategias efectivas para el aprendizaje. Desde los primeros años reconoce la multifuncionalidad que la pregunta adquiere para llevar a cabo los diferentes procesos en el aula. La experiencia le hará constatar de qué manera podría focalizar el uso de la estrategia para responder eficientemente a las exigencias académicas en las diferentes actividades curriculares. De acuerdo con Abio (2010) la formulación de preguntas en el aula puede tener diferentes focalizaciones como, por ejemplo, "de los conocimientos y saberes de los profesores, de las buenas prácticas en sala de aula, de las intenciones de los profesores o alumnos, de los conocimientos adquiridos por los alumnos con esas prácticas" (p. 3) e incluso desde una perspectiva eminentemente lingüística pues hay ciertos elementos del lenguaje que podrían motivar un tipo de pregunta $y$, al mismo tiempo, un modelo de respuesta esperable en el estudiantado.

Según refieren Costa \& Kallick (2015) los buenos docentes no solamente cuestionan en sus clases sino que lo hacen de manera intencionada. Conforme a estos autores una estrategia beneficiosa para el aprendizaje sería formular preguntas que estimulen el pensamiento de los educandos en diversos niveles cognitivos, por ejemplo, enunciar interrogantes que impliquen recordar hechos o situaciones, aplicar, predecir hasta llegar a evaluar o juzgar. Otra estrategia sería la construcción de preguntas a partir de suposiciones positivas, lo que asociaría el proceso con eventos más significativos para los aprendices y, por tanto, más permanentes y aplicables a distintos campos de acción. Desde la óptica de aprendizajes más valiosos, Melgar, Elisondo, Donolo \& Stoll (2016) postulan la necesidad de generar contextos educativos creativos que hagan posible la innovación educativa gracias a experiencias positivas para el estudiante: "emociones positivas y vivencias significativas desempeñan un papel importante en las experiencias inesperadas y ofrecen interesantes resquicios para la innovación educativa" (p. 44).

En concordancia con lo anterior, el estudiante aprende verdaderamente cuando logra comprender el sentido de lo que aprende, por lo tanto se hace necesario mantener la actividad a través de reflexiones constantes y cuestionamientos que impliquen más que una estrategia de inicio o de término de clase. Así lo postula Maarfia (2017b) al señalar que, en la interacción didáctica, la pregunta es más que un acto introductorio para iniciar o reiniciar la conversación. “Es la piedra angular de todo diálogo didáctico, de toda 
actividad pedagógica, dado que en clase nada se produce sin cuestionamiento" (p. 96i. Su razonamiento se basa en que cada profesor pregunta para ejecutar su clase, para enseñar con propósitos definidos, para reflexionar con sus estudiantes, para evaluar lo que han aprendido y para la construcción de nuevos conocimientos. En esta misma línea Colás Bravo (2009) indica que preguntar es una de las actividades más representativas de la acción didáctica en el aula puesto que, además de ser un recurso al que el profesor dedica gran parte de su tiempo, es una estrategia que puede ejercer una influencia positiva en el aprendizaje de sus estudiantes.

Los planteamientos precedentes son consistentes con Zuleta (2005), quien da cuenta de la importancia de la pregunta y de los ámbitos de acción en que puede ser un aporte significativo para el alumnado: "El uso de la pregunta es sustancial porque propicia la reflexión, el planteamiento de problemas o hipótesis. Favorece, además, la expresión oral y/o escrita, la comunicación entre estudiantes, su atención y la creación de un ambiente favorable de aprendizaje" (p. 116). Añade que la pregunta, en tanto contribución para el aprendizaje, requiere ser aprendida y enseñada en el aula. No obstante, a pesar de que el preguntar es una técnica empleada de forma espontánea, en el aula no siempre se implementa de la manera más adecuada como recurso didáctico (Van de Velde, 2014) o "muchas veces se centra en aspectos formales del lenguaje más que en el significado y el sentido" (Forero Sáenz, 2014, p. 27).

Desde la perspectiva del docente también son muchas las utilidades. De acuerdo con Maarfia (2017a) la pregunta ocupa un lugar fundamental en la interacción que se propicia entre profesor y estudiante pues brinda al docente la posibilidad de comprobar lo que se sabe, de guiar a los educandos hacia las habilidades lingüísticas y de evaluar lo que se ha comprendido. Esta retroalimentación es una condición necesaria en todo proceso de aprendizaje y en los distintos momentos de la clase: la motivación, el desarrollo y el cierre. En el primer momento permite la activación de los conocimientos previos; en el segundo posibilita la adquisición y desarrollo de nuevos conocimientos, el monitoreo, la evaluación formativa y la reflexión y en el tercero propicia la verificación de conocimientos, la evaluación integradora y la reflexión orientada hacia la mejora de los aprendizajes. Por consiguiente, el recurso de la pregunta hace posible el intercambio de saberes y el afianzamiento del conocimiento ya internalizado en el sistema cognitivo de cada estudiante.

Como se ha señalado la pregunta se concibe como una estrategia transversal para el aprendizaje de distintas disciplinas. En el área de la geografía Granados (2017) sostiene que la pregunta como estrategia didáctica no solamente contribuye en la enseñanza del área disciplinar sino en el desarrollo del pensamiento crítico de los estudiantes (p. 557). Por ello subraya la necesidad de formular preguntas que impliquen diferentes niveles cognitivos. En el ámbito de las ciencias, García González \& Furman (2014) destacan el valor de la pregunta para el docente sugiriendo la implementación de situaciones de aprendizaje en las que los estudiantes utilicen el cuestionamiento como "parte fundamental de su trayecto formativo y que los sitúe como protagonistas de la construcción de conocimiento en la escuela, contribuyendo con la creación de hábitos que favorezcan la imaginación, la creatividad y el pensamiento colectivo" (p. 89).

Otro aspecto importante que debe mencionarse es que la pregunta no solamente se da en la relación entre profesor y estudiante, en una relación diádica para aprender nuevos contenidos y reforzar los ya internalizados sino que, asimismo, se hace patente en los ambientes de trabajo colaborativo en los cuales el alumnado aprende en su relación con 
“otros" significativos. Para Vargas \& Guachetá (2012) la formulación de preguntas en un contexto de colaboración implica "la búsqueda del encuentro de experiencias para hacer viable la comprensión de lo que sucede y les sucede a quienes están presentes en una situación dialógica, por ejemplo, en un salón de clase" (p. 174). En la medida en que se potencie este tipo de aprendizaje, el estudiante dispondrá de herramientas más sólidas para enfrentar los diversos desafíos de la educación formal.

En consonancia con el logro de un aprendizaje significativo es importante mencionar que los objetivos para los que se emplea la pregunta son de diversa naturaleza. Ello no desmerece el valor que algunas tienen respecto de otras pues el conjunto de ellas podría estar en una relación de inclusión donde cada una aporta, desde una arista diferente, a la consecución de aprendizajes de valor para el estudiantado.

En el contexto de la investigación realizada la tabla 1 presenta una tipología de las diferentes finalidades para las que los docentes podrían utilizar la estrategia en el aula.

Tabla 1. Tipología de preguntas de acuerdo con su finalidad

\begin{tabular}{|l|l|}
\hline \multicolumn{1}{|c|}{ Finalidad } & \multicolumn{1}{c|}{ Habilidad cognitivo-lingüística predominante } \\
\hline Verificación de información & Definir/describir \\
\hline Fines prácticos & Explicar \\
\hline Reafirmación de la emotividad & Justificar \\
\hline Establecimiento de la comunicación & Describir/explicar/justificar \\
\hline Reflexión & Describir/explicar/justificar/argumentar \\
\hline Aprendizaje & Definir/describir/explicar/justificar/argumentar \\
\hline
\end{tabular}

Fuente: elaboración propia. Referencia para habilidades cognitivo-lingüísticas, Jorba (2000).

En consonancia con la tabla presentada es posible observar que los diversos tipos de preguntas promueven procesos cognitivos de diversa complejidad. En el caso de la utilización de la pregunta para la reflexión y el aprendizaje se vuelve necesario activar variadas habilidades cognitivo-lingüísticas.

Finalmente cabe destacar que si bien existen diferentes tipos de cuestionamientos que remiten a la dimensión pedagógica y a su rol como instrumento de evaluación, también se concibe a la pregunta "como un conjunto de prácticas sociales" (Zárate, 2015, p. 303). Este hecho conlleva entender el contexto, las ideologías, los conocimientos previos del estudiante y su capacidad para poner en práctica la metacognición.

\section{La reflexión motivada por la formulación de las preguntas en el aula}

Todo profesor espera que sus estudiantes pongan en práctica la reflexión en el aula. Concebirla en cada una de las planificaciones implica entender las diferencias entre alumnos, sus habilidades cognitivas, metacognitivas y lingüísticas, entre otras, y diseñar planes de trabajo contextualizado que permitan abordar el aprendizaje desde el conocimiento del mundo del estudiante. Los procesos reflexivos se asocian con un aprendizaje consciente, con un aprendiz involucrado en las tareas que debe resolver y con un profesor receptivo a las necesidades de cada curso. Dicha receptividad puede materializarse a través de la retroalimentación formativa en los distintos momentos de la clase, la que es posible mediante la utilización de las preguntas. Según Capilla (2016), el propósito del sistema educativo en la actualidad es "dotar al sujeto de herramientas 
que le permitan construir y reconstruir constantemente saberes diversos por sí mismo" (p. 50). Este tipo de aprendizaje puede potenciarse mediante prácticas efectivas y el desarrollo de habilidades cognitivas, motivadas por el cuestionamiento permanente.

De acuerdo con lo anterior las interrogantes formuladas en el aula van dirigidas a reflejar diferentes grados de conocimiento y a desarrollar una o más habilidades cognitivolingüísticas. Es un hecho que en años precedentes el foco de la atención estaba en la memorización y repetición de la información. Sin embargo, en la actualidad el interés se centra en el fomento de habilidades superiores de pensamiento y, por consiguiente, en el desarrollo del pensamiento crítico. Según la perspectiva del profesorado, es relevante el desarrollo y fortalecimiento de variadas habilidades cognitivo-lingüísticas en el alumnado (Jorba, 2000). Una de las habilidades que se busca fortalecer es la argumentación, considerada una habilidad de orden superior que asocia el logro de los aprendizajes con una perspectiva metacognitiva y que implica un trabajo activo y reflexivo por parte del estudiante. Por consiguiente, con su implementación en el aula el estudiantado va más allá de la recepción de contenidos y actúa como un agente crítico de sus procesos.

En su formación como futuros profesores el alumnado debe comprender la importancia de promover la reflexión mediante preguntas que lleven a cuestionar fenómenos, puntos de vista con argumentos sólidos y válidos. Conforme a los momentos de la clase, el cuestionamiento brinda la posibilidad de que los estudiantes hagan uso de su sistema cognitivo para poner en juego una serie de estrategias para el aprendizaje. Al inicio de la sesión el profesor invita a proyectar la información, a la generación de hipótesis. Por su parte, en el cierre de clase propicia la utilización de estrategias metacognitivas para valorar lo abordado en el curso así como para evaluar las dificultades experimentadas y analizar de qué manera sería posible remediarlas para mejorar los aprendizajes. Ello sustenta un trabajo con miras a la resolución de problemas y al pensamiento crítico y reflexivo a lo largo del proceso de formación.

Para la puesta en práctica de estas habilidades y estrategias es importante remarcar que los cambios tecnológicos y sociales han llevado a forjar un estudiante crítico e interactivo que requiere de un aprendizaje basado en metodologías activas, en un ambiente donde es capaz de expresar sus opiniones y cuestionar aquellos aspectos susceptibles de perfeccionar. En esta misma línea, los hallazgos de Méndez Garrido \& Conde Vélez (2018) dan cuenta de que "El alumnado demanda una metodología dinámica, participativa, práctica, amena, flexible. Parece defenderse un proceso de trabajo fundamentalmente práctico que permita la participación y un mayor nivel de protagonismo del alumnado en el aula" (p. 25). Este tipo de metodología podría implementarse mediante la utilización de preguntas coherentes con las temáticas y con los objetivos planteados en clases. La finalidad de las preguntas en dicho proceso no solamente sería una forma de inducir repuestas posibles sino un modo de aprendizaje: "el docente pregunta no sólo para activar la búsqueda de respuestas sino para enseñar a preguntar, de modo que el estudiante aprenda a autoestimularse, es decir, aprenda a aprender y con ello a desarrollar su conocimiento" (Morón, 2015, p. 5).

La autoestimulación hacia el descubrimiento de nuevos aprendizajes exige, de manera ineludible, una retroalimentación por parte de los otros significativos tales como los profesores. Es importante que el estudiante reciba la retroalimentación ya sea cuando formula preguntas en el aula como cuando responde a las interrogantes enunciadas por el profesor. Según Yang (2017) la evaluación inmediata de las preguntas y respuestas del alumnado podría garantizar la efectividad de este recurso en el aula, lo que implica que los 
docentes necesitan proveer retroalimentación a los estudiantes después de responder a las preguntas. Ello es posible con profesores comprometidos con sus prácticas al interior del aula, que utilizan tanto las buenas experiencias como las dificultades para el mejoramiento de los aprendizajes. Rojas \& Joglar (2017) sostienen que "cada vez con más frecuencia, se instala la necesidad de que las y los estudiantes formulen buenas preguntas permitiendo que éstas jueguen distintos roles, desde la construcción de saberes, hasta el desarrollo del pensamiento crítico y creativo" (p. 2).

En relación con la referida construcción del conocimiento, la enseñanza-aprendizaje a nivel reflexivo demanda que los estudiantes sean capaces de emplear estrategias cognitivas y metacognitivas para mejorar los procesos de comprensión y producción del lenguaje. Las mismas estrategias son facilitadas mediante la utilización de una estrategia pedagógica como la pregunta al momento de la activación del conocimiento previo del estudiante. Según Fasel (2013) la aplicación de un enfoque de esta naturaleza no solo crea un aprendizaje activo para el educando sino que también le da acceso a una reflexión metacognitiva sobre su manera de expresarse y de interactuar en sociedad, al tiempo que refuerza la confianza en sus capacidades (p. 29). De igual forma se requiere de profesores preparados para atender a la diversidad de formas de pensamiento de sus estudiantes, docentes competentes en el uso de estrategias para llevar con éxito sus prácticas educativas y maestros dispuestos a guiar y fomentar la reflexión en el aula. Curtet, Perini \& Tissot (2015) extreman la importancia de la pregunta como una potente herramienta didáctica para promover un enfoque reflexivo en los futuros docentes.

Resta señalar que la interacción generada entre los estudiantes hace posible un intercambio de saberes y una relación de complementariedad entre los participantes del acto comunicativo. El empleo de preguntas como estrategia didáctica favorece la reflexión en la medida en que potencia una actitud crítica frente a temáticas planteadas y hace posible la construcción de significados relevantes para el estudiante. Del mismo modo, al establecerse como una estrategia que guía el proceso puede derivar en aprendizajes permanentes y significativos para su formación. Finalmente, considerando su valor como estrategia transversal a las diferentes áreas de formación académica, insta a la reflexión, un proceso que juega un papel central para el mejoramiento del desempeño en el aula. Su internalización como estrategia inherente al proceso de enseñanza-aprendizaje irá en directo beneficio de las habilidades cognitivo-lingüísticas, especialmente de la argumentación, tan necesaria para la creación de significados y para el asentamiento de una postura individual que colabora en la definición de la identidad de cada persona.

\section{Metodología}

La presente investigación se aproxima a las percepciones que tienen estudiantes de pedagogía acerca de la pregunta y la analiza como una estrategia pedagógica para abordar el subsector de lenguaje y comunicación, específicamente en lo referente a la reflexión que la pregunta puede suscitar en el aula cuando se aborda una temática particular.

El diseño metodológico fue de carácter mixto, con un enfoque descriptivo (Hernández, Fernández \& Baptista, 2014). Para la recolección de información se elaboró un cuestionario en línea conformado por preguntas tanto abiertas como cerradas (Corral, 2010). En concordancia con la bibliografía consultada y los objetivos del investigador los criterios para la incorporación de los cuestionamientos en el instrumento tienen relación con la obtención de información sobre los siguientes tópicos: 

a) Habilidades cognitivo-lingüísticas activadas por la pregunta como estrategia didáctica;
b) Habilidades cognitivo-lingüísticas reforzadas por la pregunta como estrategia;
c) Finalidad con que los docentes en aula emplean la estrategia de cuestionar;
d) Términos asociados con el uso de la estrategia, y
e) Propósitos por los cuales los estudiantes de pedagogía emplean la pregunta en sus prácticas en establecimientos de enseñanza primaria y secundaria.

El proceso de construcción del cuestionario consideró dos etapas:

1) Un estudio normativo aplicado a 50 estudiantes de diferentes carr eras de pedagogía, cuyo objetivo fue determinar el índice de aceptabilidad de las interrogantes y enunciados. De una escala de 1 a 5, donde 1 era lo menos aceptable y 5 completamente aceptable, se seleccionaron solamente aquellas preguntas con el nivel de aceptabilidad más alto (4 y 5). Las respuestas a las mismas se ven reflejadas en los resultados del presente estudio.

2) Un juicio de expertos a tres profesores universitarios, a fin de recoger comentarios sobre el instrumento y proceder a la realización de los ajustes necesarios antes de la aplicación formal.

Con la información obtenida a partir de las respuestas de los informantes fue posible realizar dos tipos de análisis: uno cualitativo realizado a partir del corpus textual emergente de las respuestas abiertas, bajo el enfoque de la Teoría Fundamentada y el uso del Método Comparativo Constante (Vieytes, 2004; Strauss \& Corbin, 2002), y otro de tipo cuantitativo elaborado a través de un Análisis Descriptivo: en concreto, el análisis de frecuencia absoluta.

\section{Objetivos}

El objetivo general de la investigación fue analizar la importancia de la pregunta como estrategia didáctica y de reflexión para futuros profesores de lenguaje. Por su parte, los objetivos específicos fueron los siguientes:

1. Determinar las habilidades que las preguntas potencian en el profesorado en formación. 2. Identificar las habilidades que a juicio de los participantes activa la pregunta en el trabajo en aula.

3. Analizar los fines para los cuales la pregunta es más idónea de abordar en el subsector de lenguaje y comunicación.

\section{Participantes}

Participaron del estudio cuarenta estudiantes de pedagogía del subsector Lenguaje y Comunicación que cursan su tercer año en una universidad chilena. La edad promedio del grupo fue de 21 años. La muestra se define como no probabilística intencionada dado que fue el propio investigador quien seleccionó intencionalmente a los sujetos que participaron en la investigación (Albert, 2007; Otzen \& Manterola, 2017; Hernández, Fernández \& Baptista, 2014).

\section{Procedimiento}

Las técnicas de recolección de datos corresponden a las respuestas generadas mediante el cuestionario en línea con preguntas abiertas y cerradas. Las fases de trabajo se llevaron a cabo durante un semestre lectivo. Los contenidos de la asignatura están relacionados con la didáctica del lenguaje, especialmente en el área de la comprensión. A lo largo del semestre se abordó la pregunta como estrategia para la comprensión y la producción 
de discursos. Un mes antes del término del semestre se solicitó a los estudiantes de pedagogía participantes responder el cuestionario, cuya información se trabajó de manera confidencial. No todos los estudiantes, pertenecientes a dos secciones diferentes, respondieron a dicho cuestionario.

\section{Resultados}

Con el propósito de estudiar la pregunta como estrategia didáctica el análisis de los datos se focalizó en la identificación de las habilidades que las preguntas potencian y activan en el trabajo en aula, y en la relevancia para el trabajo en la especialización de lenguaje y comunicación.

El cuestionario aplicado permitió conocer qué habilidades lingüístico-cognitivas se movilizan al momento de formular una pregunta en clase. De acuerdo con el proceso de recolección los informantes responden desde su posición de estudiantes de una carrera universitaria de pedagogía y, al mismo tiempo, desde la perspectiva de futuro profesor. A continuación se da cuenta de los porcentajes obtenidos por el grupo en cada una de las habilidades fundamentales para los estudiantes de lenguaje.

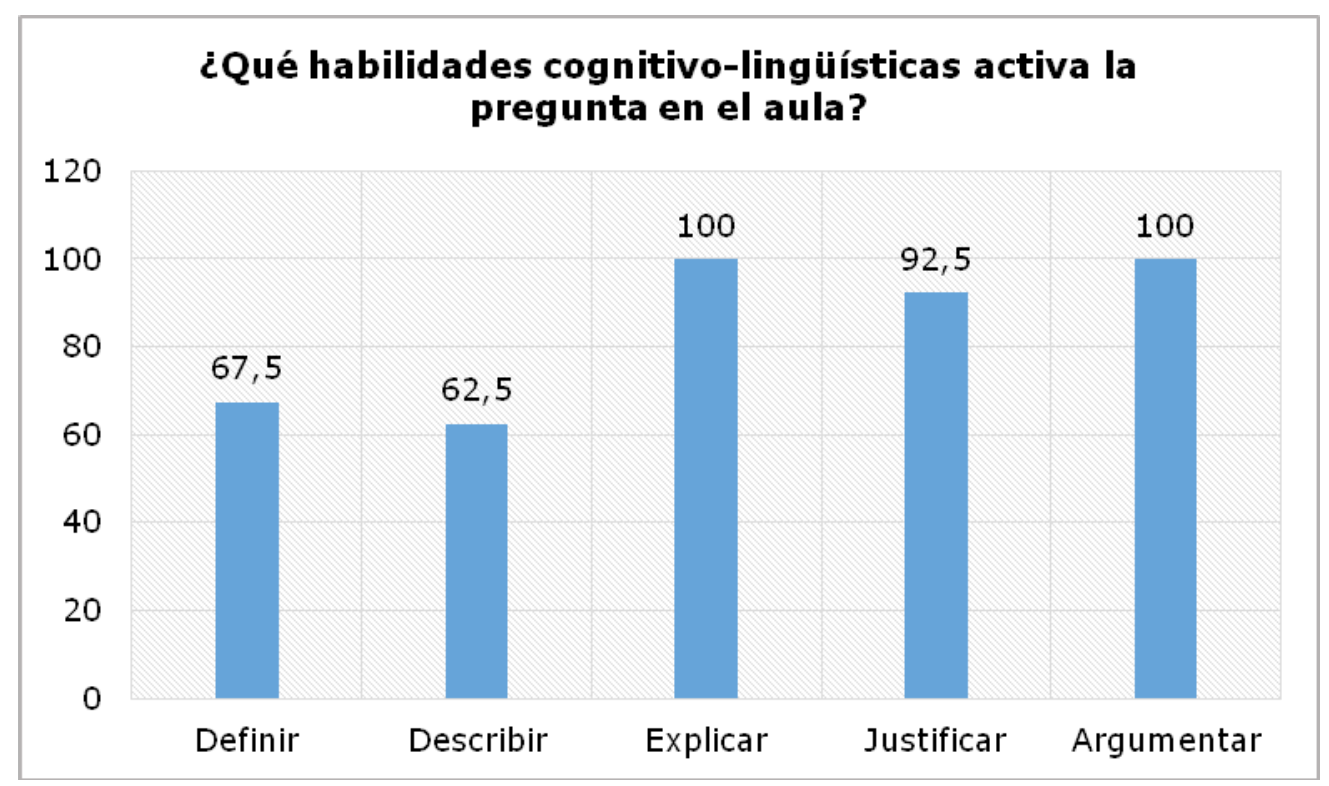

Figura 1. Porcentaje de respuesta sobre todas las habilidades activadas por la pregunta

La figura 1 evidencia la percepción que poseen los informantes acerca de todas las habilidades lingüístico-cognitivas que la pregunta, como estrategia, activa en el aula. De acuerdo con los resultados, el $100 \%$ de los participantes considera que tanto la habilidad de argumentar como la de explicar están íntimamente relacionadas con el uso de la pregunta durante el trabajo académico. Por su parte, las otras habilidades también se accionan dependiendo de la finalidad con la que se aborde la pregunta en clase.

Ahora, frente a la elección de una sola alternativa, los resultados suelen ser similares en aquellas habilidades que presentan un más alto porcentaje de valoración. Como se reconoce en educación, una de las habilidades que más buscan reforzar los docentes en el alumnado es la argumentación, independientemente del nivel de escolarización y de la carrera universitaria que cursa cada estudiante. 


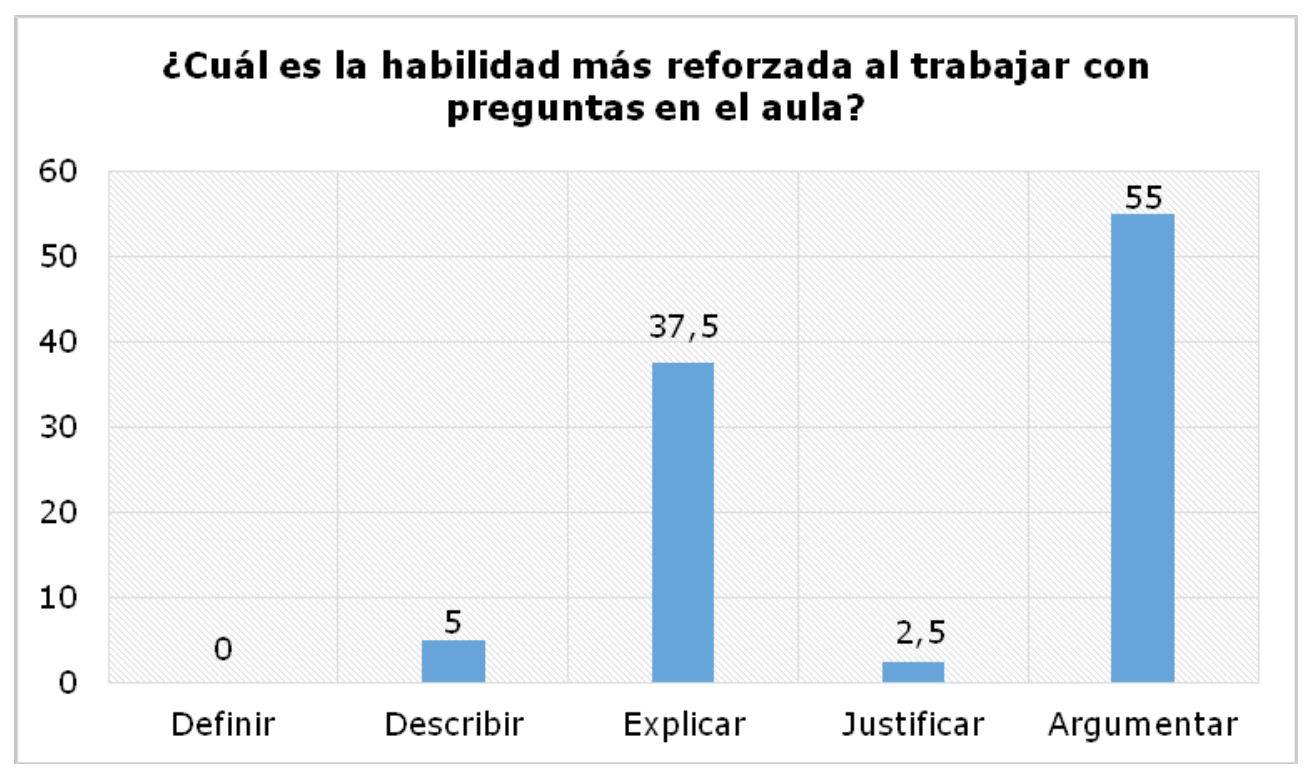

Figura 2. Porcentaje de respuesta sobre habilidad más reforzada por la pregunta

La figura 2 muestra que el 55\% del estudiantado participante reconoce que la pregunta favorece la capacidad de argumentar y el $37,5 \%$ señala que refuerza el explicar. Esta última es una habilidad básica y fundamental para los docentes pues en cada acto comunicativo y didáctico el profesor debe explicar a sus estudiantes. De la misma forma los alumnos deben explicar conceptos, teorías y aprendizajes, entre otros. Por ello se considera que la formulación de preguntas media como estrategia en una relación bidireccional: el estudiante cuestiona cuando tiene dudas acerca de un tema y el profesor, por su parte, pregunta cuando quiere verificar información o el aprendizaje frente a una temática específica.

Con la idea de profundizar en dicha información se solicitó a los informantes justificar la respuesta sobre las habilidades más afianzadas en los estudiantes en el aula. Debido a la diversidad de respuestas y a la extensión de las mismas se presentan fragmentos de discurso seleccionados de manera aleatoria. A fin de resguardar la confidencialidad de los participantes se utiliza la abreviación E ("estudiante") con el número empleado para efectos de codificación.

Tabla 2. Discurso relacionado con la pregunta como estrategia para la explicación.

\begin{tabular}{|c|l|}
\hline Código & Respuestas \\
\hline E07 & $\begin{array}{l}\text { "La pregunta mejora la capacidad para explicar porque comúnmente los } \\
\text { estudiantes no se dan el trabajo de desarrollar sus ideas". }\end{array}$ \\
\hline E12 & "A través de la pregunta el estudiante puede explicar su punto de vista". \\
\hline E18 & $\begin{array}{l}\text { "Las preguntas que los estudiantes hacen en el aula al profesor son para pedir } \\
\text { la explicación de algún concepto que no ha quedado claro o en el caso del } \\
\text { profesor que pregunta a los alumnos, mayoritariamente es para saber si el } \\
\text { estudiante puede explicar lo que se ha enseñado". }\end{array}$ \\
\hline
\end{tabular}

Fuente: elaboración propia. 
En los ejemplos exhibidos en la tabla 2 se advierte que la pregunta desempeña un papel fundamental a la hora de explicar aspectos de significación para la clase. Concretamente, los informantes destacan la importancia de la explicación y la relacionan de manera directa con la labor del profesor, con la clarificación de ideas o conceptos y con la consolidación de conocimientos. Por su parte, los discursos que se exponen a continuación muestran una valoración de la pregunta como una estrategia para fomentar la capacidad de argumentar.

Tabla 3. Discurso relacionado con la pregunta como estrategia para la argumentación.

\begin{tabular}{|c|l|}
\hline Código & Respuestas \\
\hline E27 & $\begin{array}{l}\text { "La pregunta en el aula podría activar todas las habilidades cognitivo- } \\
\text { lingüísticas, en especial la argumentación, porque es una estrategia } \\
\text { transversal a los diferentes contenidos de la carrera" }\end{array}$ \\
\hline E37 & $\begin{array}{l}\text { "La habilidad de argumentar, que es de mayor complejidad y puede incluir } \\
\text { todas las anteriores, se refuerza por la pregunta a la hora de apropiarse del } \\
\text { conocimiento y del aprendizaje". }\end{array}$ \\
\hline E40 & $\begin{array}{l}\text { "Cuando el estudiante argumenta su respuesta demuestra sus conocimientos, } \\
\text { le permite defender su postura y obtener seguridad". }\end{array}$ \\
\hline
\end{tabular}

Fuente: elaboración propia.

Los casos presentados plasman una relación estrecha entre la utilización de la pregunta y la necesidad de que los estudiantes desarrollen la capacidad de defender sus ideas y puntos de vista. Asimismo se hace mención a la complejidad de unas habilidades en comparación con otras. Esta noción se complementa con la existencia de una transversalidad en el uso de la pregunta, que permite activar diferentes habilidades y, al mismo tiempo, responder a los distintos requerimientos propios de una actividad curricular.

Con el propósito de ahondar aún más en la utilización de la pregunta como estrategia pedagógica se buscó determinar, a través de un cuestionamiento de tipo cerrado, con qué finalidad el docente utiliza más habitualmente la pregunta en el aula de enseñanza media. 


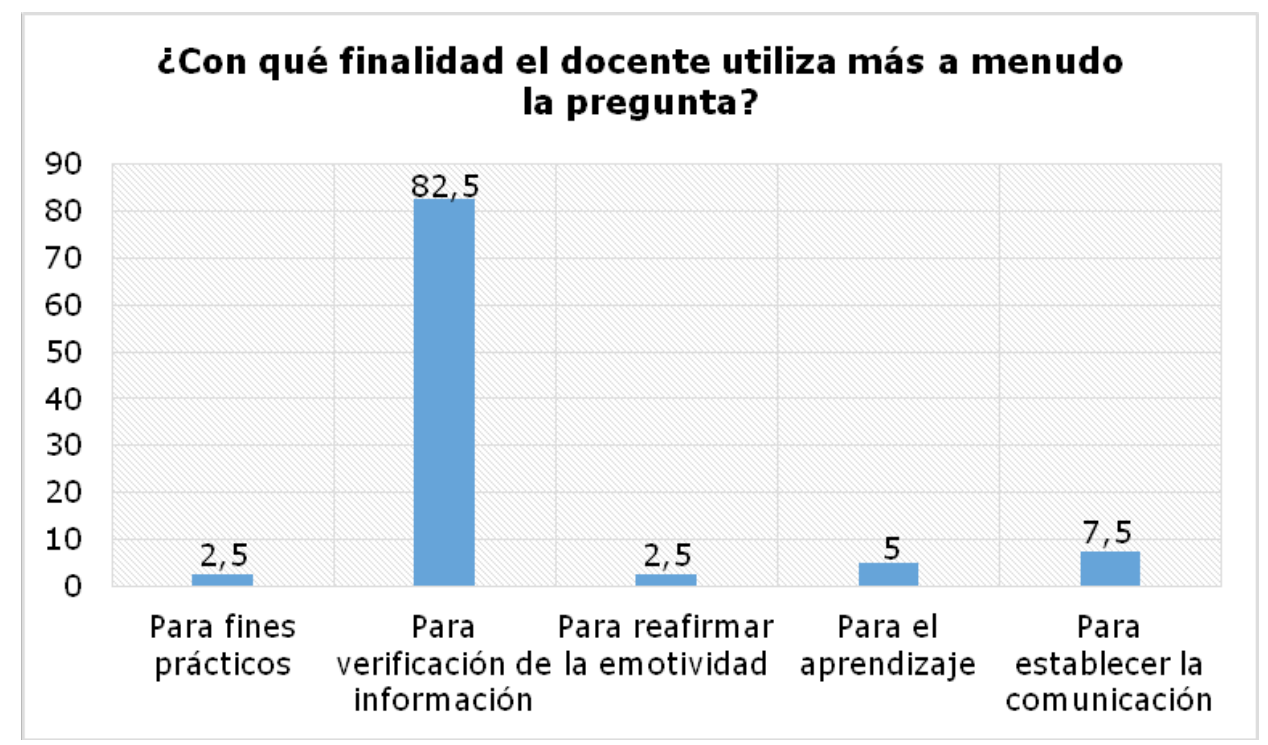

Figura 3. Porcentaje de respuesta sobre finalidad para utilización de la pregunta.

La figura 3 refleja que los docentes emplean más frecuentemente la estrategia de la pregunta como una forma de verificación de información; en concreto, el 82,5\% manifiesta esta elección. Tales respuestas son complementadas por el análisis cualitativo del discurso de los participantes. En una pregunta abierta se les solicita que señalen, desde su papel de futuro educador (a), las tres palabras que asocian al uso de la pregunta en el aula. Al analizar la primera de dichas palabras se obtienen las siguientes frecuencias.

Tabla 4. Términos asociados a la estrategia de formulación de preguntas.

\begin{tabular}{|l|l|l|}
\hline \multicolumn{1}{|c|}{ Términos } & \multicolumn{1}{|c|}{ Frecuencia } & \\
\hline Reflexión & 12 & 30 \\
\hline Aprendizaje & 8 & 20 \\
\hline Conocimiento & 7 & 17,5 \\
\hline Pensamiento crítico & 6 & 15 \\
\hline Metacognición & 5 & 12,5 \\
\hline Argumentación & 2 & 5 \\
\hline
\end{tabular}

Fuente: elaboración propia. 
Según la tabla, 12 estudiantes de pedagogía (30\%) sostienen que la pregunta se asocia a la reflexión en el aula. Por su parte, el 20\% la asocia al aprendizaje y el 17,5\% al conocimiento. Otras palabras mencionadas (en segundo y tercer lugar) son: asimilación, ayuda, coevaluación, cognición, comprensión, comunicación, contexto, diálogo, duda, ejemplificación, estimulación, evaluación, evidencia, interacción, interpretación, juicio, monitoreo, objetividad, observación, opinión, percepción, proceso, resolución, retroalimentación, transformación y verificación. Dentro de los términos mencionados en segundo y tercer lugar los citados con mayor frecuencia fueron monitoreo y retroalimentación, los que habitualmente se relacionan con la evaluación y cierre de una clase.

\section{Discusión}

La formulación de preguntas en el aula favorece el intercambio comunicativo y la integración de saberes por parte del alumnado. De acuerdo con los hallazgos de esta investigación los informantes consideran de gran relevancia la pregunta dado que fomenta las habilidades cognitivo-lingüísticas referidas a la explicación y a la argumentación. Si bien el uso de la estrategia podría asociarse con una tarea cotidiana, implica un pensamiento crítico y reflexivo de los estudiantes tal como señalan González \& Castro (2011), quienes afirman que aprender a preguntar no se realiza de manera automática sino que requiere de la intervención del profesor para dirigir y monitorear los procesos involucrados. La implementación de la estrategia necesita "una estructura didáctica que lleve a los alumnos a otros niveles de pensamiento" (p.59) en los que pueda tomar decisiones y resolver situaciones problemáticas.

Los datos presentados realzan la importancia de la pregunta como estrategia para ser utilizada en los distintos momentos de la clase, hallazgo consistente con Prabowo \& Alfiyanti (2013) quienes ponen énfasis en el valor del cuestionamiento como una herramienta para motivar el interés de los estudiantes por un tema en específico, para activar los conocimientos previos y para cumplir con los propósitos definidos por el docente.

Otro hallazgo importante se relaciona con el hecho de que si bien a juicio de los informantes la pregunta surge como una estrategia para el desarrollo del pensamiento crítico y de la reflexión, el profesorado de enseñanza media formula preguntas esencialmente para practicar la memorización y la verificación de información.

Desde una perspectiva eminentemente cualitativa se analiza el discurso derivado del cuestionamiento: “Desde su rol como futuro educador, ¿con qué propósito usaría la pregunta en el aula?". Con las respuestas y el consiguiente análisis del contenido temático (Gibbs, 2012) se definieron cuatro categorías principales que no son mutuamente excluyentes:

1. La pregunta potencia una actitud crítica.

2. La pregunta permite la construcción de aprendizajes.

3. La pregunta fomenta el autoaprendizaje.

4. La pregunta genera reflexión para la mejora del desempeño en aula. 


\section{La pregunta potencia una actitud crítica}

En consonancia con las respuestas obtenidas se constata en los estudiantes de pedagogía una valoración de la pregunta en tanto promueve una actitud crítica frente a los aprendizajes y a la manera de evaluar los conocimientos adquiridos.

Un informante indica que: “Utilizaría la interrogante para verificar que los estudiantes presenten claridad sobre el tema enseñado y que sean capaces de llevar su conocimiento a una habilidad superior, como el argumentar" (estudiante 9).

Otro informante señala que el uso de la estrategia le permitiría "reforzar habilidades cognitivas en el estudiante y, de este modo, adoptar una actitud crítica" (estudiante 15).

Esta misma idea es recogida en el siguiente discurso por otro informante (estudiante 21): “Implementaría la estrategia para evaluar el aprendizaje de los estudiantes y verificar la comprensión de los contenidos trabajados, así como para activar los conocimientos previos y lograr que reflexionen sobre un tema. Fuera del aula se puede utilizar con el fin de obtener nueva información de interés y, asimismo, reflexionar y generar opiniones críticas".

\section{La pregunta como construcción de aprendizajes}

La pregunta contribuye al proceso de enseñanza-aprendizaje en la medida en que los aprendices son capaces de construir sentidos (Ausubel, 2009). Ese es, precisamente, uno de los hallazgos del estudio. Los futuros docentes declaran que la pregunta les permite retroalimentar sus propios procesos al interior del aula y, al mismo tiempo, les brinda la posibilidad de instalarse en sus centros de práctica con una herramienta efectiva para el logro de los aprendizajes del estudiantado.

La idea antes referida es consistente con Döş et al. (2016) quienes postulan que la interrogación contribuye especialmente a la enseñanza efectiva de todos los educandos, lo que es posible en la medida en que exista una inmersión en el conocimiento de cada agente involucrado en el proceso. Uno de los estudiantes de pedagogía indica que utilizaría la pregunta para asegurarse "de que los estudiantes se encuentren aprendiendo sobre alguna temática y saber qué es lo que debe reforzarse en cuanto a contenidos", es decir, como una estrategia para ir consolidando los aprendizajes y reforzando aquellos más deficitarios. Dicha construcción de aprendizajes es mediada por el diálogo entre los estudiantes y con el profesor, lo que hace posible mantener activa la clase.

\section{La pregunta como fomento del autoaprendizaje}

En apoyo a los postulados de Maarfia (2017b) la pregunta como estrategia pedagógica fomenta el autoaprendizaje dado que es una estrategia flexible que puede ser utilizada tanto por el profesor como por los estudiantes en distintos momentos del proceso de enseñanza. En este contexto los informantes expresan que la estrategia puede ser implementada como "método de verificación de aprendizaje" (estudiante 16) y, a su vez, como un recurso que les permitiría "ser protagonistas de su aprendizaje" (estudiante 11), confirmando lo referido por Morón (2015) acerca de que esta estrategia constituye uno de los medios más eficaces para favorecer el proceso de enseñanza-aprendizaje. Se subraya el hecho de que el aprendiz debe responsabilizarse de su proceso y asumir el compromiso de aplicar las estrategias necesarias para afianzar sus conocimientos.

Otro de los informantes (estudiante 25) manifiesta que, "la utilizaría como preguntas guiadas que lleven a los alumnos a descubrir por sí mismos el aprendizaje, así como para verificar cómo están adquiriendo los conocimientos y, por último, para impulsarlos a desarrollar sus habilidades comunicativas generando respuestas inmediatas cada vez más eficaces de sus propias interpretaciones". 


\section{La pregunta genera reflexión para la mejora del desempeño en aula}

En consonancia con lo que afirman González \& Castro (2011) los resultados revelan que la pregunta podría motivar la reflexión puesto que brinda al estudiante la posibilidad de ir monitoreando su propio aprendizaje y de analizar aquellos aspectos más significativos del mismo. La mayoría de los informantes declara que la pregunta permite consolidar conocimientos y constatar qué se debe reforzar. Asimismo señalan que la pregunta podría motivar la reflexión durante la clase o después de una actividad, lo que implicaría instancias para asentar contenidos, desarrollar habilidades y reforzar nuevos conocimientos que, consecuentemente, podrían ser vinculados con el diario vivir de cada estudiante y aplicados en circunstancias reales.

Mientras para unos informantes la formulación de preguntas se asocia directamente con la resolución de problemas, para otros se relaciona con el desarrollo de habilidades cognitivo-lingüísticas tales como el argumentar, lo que deviene en una motivación por profundizar aún más en los aprendizajes que ya se han internalizado. Uno de los discursos (estudiante 30) establece que “El responder una pregunta requiere de una reflexión, sobre todo si se quiere argumentar. El profesor puede pedir al alumno desarrollar más la respuesta, o que entre toda la clase se elabore una respuesta más completa. Si se presenta algún error el profesor los va guiando por medio del diálogo".

\section{Conclusiones}

Como estrategia pedagógica la pregunta requiere sofisticación en su uso y demanda una planificación detallada sobre la forma de implementarse en el aula. En este contexto Maarfia (2017a) sostiene que los docentes deben estar atentos a ciertos aspectos de su actividad de cuestionamiento, a saber, la longitud de las preguntas, la velocidad de su secuencia, las situaciones fuera de la clase a las que se refieren $y$, finalmente, su adecuación con el nivel de producción de los estudiantes. Dado que los maestros pasan gran parte de su jornada escolar haciendo preguntas, Joseph (2018) señala que es importante que dediquen tiempo a planificar los cuestionamientos específicos para alentar la discusión y desafiar el pensamiento crítico. Se recomienda pensar en la adaptabilidad de las preguntas, implementando estrategias interrogativas que permitan a los estudiantes comprender mejor y ponerlos en una situación de profundización del conocimiento y de su capacidad reflexiva.

Si bien existen diferentes tipologías de preguntas, la mayor parte de ellas plantean como ideal el desarrollo del pensamiento crítico y de la reflexión. No obstante, según declaran los informantes del estudio, los docentes formulan las preguntas, prioritariamente para ejercitar la memorización y la verificación de información aunque reconocen los esfuerzos por el desarrollo de la argumentación en el estudiantado. En virtud de lo presentado, las principales conclusiones que derivan de la presente investigación son las siguientes:

En primer lugar, las habilidades que según el alumnado de pedagogía potencian las preguntas son el argumentar y el explicar. Por su parte, las otras habilidades (como el definir, describir y justificar) también se activan dependiendo de la finalidad con la que se aborde la pregunta en clases.

En segundo lugar, una de las habilidades que más busca reforzar el profesorado en sus estudiantes es la argumentación, independientemente del nivel de escolarización o de la carrera universitaria. Según el estudio la pregunta favorece la capacidad de argumentar y la habilidad de explicar, habilidad básica y fundamental para el estudiantado de pedagogía en sus prácticas cotidianas. 
En tercer lugar, aunque existen diferentes ámbitos de acción de la pregunta, la finalidad con la que los docentes de educación primaria y secundaria emplean con mayor frecuencia esta estrategia es la verificación de información. Este dato parece contradecir los planteamientos del estudiantado de pedagogía, quienes relevan la importancia de esta estrategia pedagógica para desarrollar la reflexión y el pensamiento crítico.

Como cuarta conclusión, la formulación de preguntas se presenta como una estrategia cimentada en una relación bidireccional pues el estudiante cuestiona cuando tiene dudas acerca de un tópico particular y el docente, por su parte, pregunta cuando quiere verificar información o el aprendizaje de sus estudiantes.

Finalmente, para los estudiantes de pedagogía participantes, la pregunta se asocia directamente con la reflexión en el aula y con la adquisición de nuevos aprendizajes significativos para la formación académica del alumnado, independientemente de su clasificación, al tiempo que potencia una actitud crítica y fomenta el autoaprendizaje, aspectos fundamentales para el asentamiento de saberes permanentes en el tiempo.

\section{Referencias bibliográficas}

Abio, G. (2010). Algunas reflexiones sobre la realización de preguntas por profesores de lenguas en formación. MarcoELE revista de didáctica ELE, 10, 1-19. https://marcoele.com/ descargas/10/abio preguntas-profesores.pdf

Albert, M. J. (2007). La investigación educativa. Madrid: Claves teóricas. https://www. academia.edu/27287685/La_Investigaci\%C3\%B3n_Educativa_Claves_Te\%C3\%B3ricas_Albert_G

Arnoux, E., Nogueira, S., \& Silvestri, A. (2007). Habilidades metacomprensivas en estudiantes de profesorado: la formulación de preguntas. Revista Folios (25), 81-95. https://doi.org/10.17227/01234870.25folios81.95

Asensio-Muñoz, I. \& Ruiz de Miguel, C. (2017). Medida y evaluación de las creencias sobre la profesión de los maestros en formación. Revista Electrónica Interuniversitaria de Formación del Profesorado, 20(3), 79-91. https://doi.org/10.6018/reifop.20.3.265231

Ausubel D., Novak J., \& Hanesian H. (2009). Psicología Educativa, un punto de vista cognoscitivo. México: Editorial Trilla.

Caira Rojas, J., Urdaneta, E. \& Mata Guevara, L. (2014). Estrategias para el aprendizaje significativo de procesos de fabricación mediante orientación constructivista.Opción,30(75), 92-103. ISSN: 1012-1587. https://www.redalyc.org/articulo.oa?id=310/31035400006

Capilla, R. (2016). Habilidades cognitivas y aprendizaje significativo de la adición y sustracción de fracciones comunes. Cuadernos de Investigación Educativa, 7(2), 49-62. https://doi.org/10.18861/cied.2016.7.2.2610

Colás-Bravo, M. (2009). La formulación de preguntas en el acto didáctico: un estudio comparativo. Enseñanza \& Teaching: Revista Interuniversitaria de Didáctica [Internet]. https://revistas.usal.es/index.php/0212-5374/article/view/3151

Corral, Y. (2010). Diseño de cuestionarios para recolección de datos. Revista Ciencias de la Educación, 20(36), 152-168. https://studylib.es/doc/5403092/dise\%C3\%B1o-decuestionarios-para-recolecci\%C3\%B3n-de-datos 
Costa, A. \& Kallick, B. (2015). Five strategies for questionning with intention. Educational Leadership, 73(1), 66-69. https://www.nwrpdp.com/uploads/1/2/3/3/123306057/5 strategies_for_questioning_with_intention_el.pdf

Curtet, L., Perini, D., \& Tissot, S. (2015). Le questionnement : proposition d'un outil d'analyse d'une pratique ordinaire. Formation et pratiques d'enseignement en questions, 18, 61-76. http://revuedeshep.ch/pdf/18/18-04-Curtet-Perini-Tissot.pdf

Döş, B., Bay, E., Aslansoy, C., Tiryaki, B., Çetin, N., \& Duman, C. (2016). An analysis of teachers' questioning strategies. Educational Research and Review, 11(22), 2065-2078. doi: 10.5897/ERR2016.3014, https://academicjournals.org/journal/ERR/article-full-text-pdf/ A09F37161683

Eshach, H., Dor-Ziderman, Y., \& Yefroimsky, Y. (2014). Question Asking in the Science Classroom: Teacher Attitudes and Practices. J Sci Educ Technol, 23, 67-81. doi: 10.1007/ s10956-013-9451-y, https://www.academia.edu/15726699/Question_Asking in the Science_Classroom_Teacher_Attitudes_and_Practices

Fasel, S. (2013). L'utilisation des stratégies de communication lors d'interactions orales en langue étrangère. HEP VAUD - Travail de mémoire, Master en Enseignement Secondaire II. https://www.patrinum.ch/record/17001

Forero Sáenz, A. (2014). El uso de las preguntas por parte del docente en la clase de matemáticas y sus efectos en las respuestas y conversaciones de los niños. Tesis doctoral, Universidad Autónoma de Barcelona, Barcelona. https://www.tdx.cat/bitstream/ handle/10803/283542/afs1de1.pdf?sequence=1

García González, S. \& Furman, M. (2014). Categorización de preguntas formuladas antes y después de la enseñanza por indagación. Praxis \& Saber, 5(10), 75-91. https://doi. org/10.19053/22160159.3023

García, S. \& Martínez, C. (2014). La importancia de las habilidades cognitivo-lingüísticas asociadas al estudio de la Astronomía desde la perspectiva del profesorado. Enseñanza de las ciencias, 32(1), 179-197. https://ddd.uab.cat/pub/edlc/edlc_a2014v32n1/edlc a2014v32n1p179.pdf

Gibbs, G. (2012). El análisis de datos cualitativos en investigación cualitativa. Madrid: Morata. https://dpp2016blog.files.wordpress.com/2016/08/graham-gibbs-el-anc3a1lisis-dedatos-cualitativos-en-investigacic3b3n-cualitativa.pdf

González, M. \& Castro, A. (2011). Impacto del ABP en el desarrollo de la habilidad para formular preguntas de aprendizaje en estudiantes universitarios. Revista de Docencia Universitaria, 9(1), 57-66. https://polipapers.upv.es/index.php/REDU/article/ view/6180/6230

Granados, J. (2017). La formulación de buenas preguntas en didáctica de la geografía. Documents d'Anàlisi Geogràfica, 63(3), 545-559. https://ddd.uab.cat/pub/dag/dag_ a2017m9-12v63n3/dag_a2017v63n3p545.pdf 
Hernández, R., Fernández, C., \& Baptista, L. (2014). Metodología de la investigación. México: Mc Graw Hill. https://www.uv.mx/personal/cbustamante/files/2011/06/Metodologia-dela-Investigaci\%C3\%83\%C2\%B3n_Sampieri.pdf

Jorba, J. (2000). La comunicación y las habilidades cognitivo-lingüísticas. En J. Jorba, I. Gómez y Á. Prats (Eds.), Hablar y escribir para aprender: uso de la lengua en situación de enseñanza-aprendizaje desde las áreas curriculares, pp. 29-49. Madrid: Síntesis. https:// es.scribd.com/doc/62536729/Jorba-2000-Hablar-y-Escribir-Para-Aprender-Copia-confines-academicos

Joseph, S. (2018). Questions Teachers Ask: An Exploratory Study of Teachers' Approach to Questioning in the Primary and Secondary Classroom. Journal of Education \& Social Policy, 5(1), 77-87. http://jespnet.com/journals/Vol5_No_1_March_2018/9.pdf

López Aymes, G. (2012). Pensamiento crítico en el aula. Docencia e Investigación, 37(22), 41-60. https://www.educacion.to.uclm.es/pdf/revistaDI/3_22_2012.pdf

López, S., Veit, E. \& Solano, I. (2014). La formulación de preguntas en el aula de clase: una evidencia de aprendizaje significativo crítico. Ciência \& Educação, 20(1), 117-132. https:// doi.org/10.1590/1516-731320140010007

Maarfia, N. (2017a). De l'usage (in)efficace du questionnement par l'enseignant en classe de FLE au primaire. Recherches en didactique des langues et des cultures, 14(2), 1-13. doi: 10.4000/rdlc.1910 https://journals.openedition.org/rdlc/1910

Maarfia, N. (2017b). Pour une didactisation du questionnement en classe de langue étrangère. Synergies Chili, 13, 95-106. https://www.researchgate.net/profile/Nabila Maarfia/publication/323587635_Pour_une_didactisation_du_questionnement_en_ classe_de langue_etrangere/links/5b601e08458515c4b25475b3/Pour-une-didactisationdu-questionnement-en-classe-de-langue-etrangere.pdf

Melgar, M., Elisondo, R., Donolo, D., \& Stoll, R. (2016). El poder educativo de lo inesperado. Estudio de experiencias innovadoras en la universidad. Cuadernos de Investigación Educativa 7(2), 31-47. https://revistas.ort.edu.uy/cuadernos-de-investigacion-educativa/article/ view/2609/2592

Méndez Garrido, J. \& Conde Vélez, S. (2018). La autorreflexión inicial: una estrategia para la mejora de la práctica docente. Revista Electrónica Interuniversitaria de Formación del Profesorado, 21(1), 17-31. doi: http://dx.doi.org/10.6018/reifop.21.1.270591

Morón, F. (2015). La importancia de hacer buenas preguntas a nuestros alumnos de la ESO. Revista Arista Digital, 54, 1-12. http://www.afapna.es/web/aristadigital/archivos revista/2015_marzo_5.pdf

Otzen, T. \& Manterola, C. (2017). Técnicas de muestreo sobre una población a estudio. Int. J. Morphol., 35(1), 227-232. https://dx.doi.org/10.4067/S0717-95022017000100037 
Prabowo, A. \& Alfiyanti, K. (2013). An analysis of teachers' questioning strategies during interaction in the classroom: a case of the eight grade SMP PGRI 01 Semarang. ETERNAL (English Teaching Journal), 4(1), 40-53. Doi: 10.26877/eternal.v4i1.1945 https:// pdfs.semanticscholar.org/e5b4/4cc71b3a6237ef76c7e741ff6425082b7292.pdf? ga $=2.25147514 .1175084062 .1594820657-535491307.1593890778$

Rojas, A. \& Joglar, C. (2017). Promoviendo buenas preguntas en la clase de ciencias a partir de situaciones problema. XI Encontro Nacional de Pesquisa em Educação em Ciências - XI ENPEC, Universidade Federal de Santa Catarina, Florianópolis, SC - 3 a 6 de julho de 2017. http://www.abrapecnet.org.br/enpec/xi-enpec/anais/resumos/R1318-1.pdf

Strauss, A. \& Corbin, J. (2002). Bases de la investigación cualitativa. Técnicas y procedimientos para desarrollar la teoría fundamentada. Universidad de Antioquia: Colombia. https:// diversidadlocal.files.wordpress.com/2012/09/bases-investigacion-cualitativa.pdf

Van de Velde, H. (2014). Aprender a preguntar, preguntar para aprender. ¿Cómo lo hacemos para aprovechar al máximo la pregunta como recurso pedagógico-didáctico? ABACOenRED. Aprendizajes Basados en Actitudes Cooperativas. https://www.ugel05.gob.pe/ documentos/10_Saber_Preguntar_Vandevelde.pdf

Vargas, G. \& Guachetá, E. (2012). La pregunta como dispositivo pedagógico. Itinerario Educativo, 26(60), 173-191. https://dialnet.unirioja.es/servlet/articulo?codigo=6280194

Vieytes, R. (2004). Metodología de la Investigación en organizaciones, mercado y sociedad. Buenos Aires: Editorial de las Ciencias. https://es.scribd.com/doc/97493378/Metodologiade-la-investigacion-en-organizaciones-mercado-y-sociedad

Yang, H. (2017). A Research on the Effective Questioning Strategies in Class. Science Journal of Education, 5(4), 158-163. doi: 10.11648/j.sjedu.20170504.16 http://spgjedu.com/ article/197/10.11648.j.sjedu.20170504.16

Zárate, A. (2015). El uso de las preguntas de comprensión crítica en los libros de texto. Foro de Educación, 13(19), 297-326. doi: http://dx.doi.org/10.14516/fde.2015.013.019.013

Zuleta, O. (2005). La pedagogía de la pregunta. Una contribución para el aprendizaje. Educere, 9(28), 115-119.

\section{(cc) BY}

Este artículo está sujeto a una licencia internacional Creative Commons Attribution 4.0.

\footnotetext{
* Universidad Católica de la Santísima Concepción (Chile)

1 Claudine Glenda Benoit Ríos Doctora en Lingüística, Magíster en Lingüística, Licenciada en Educación y Profesora de Español Universidad de Concepción (Chile). Académica, departamento de Didáctica, Facultad de Educación, Universidad Católica de la Santísima Concepción (Chile). Investigadora en procesos de comprensión y producción del lenguaje y estrategias colaborativas durante el procesamiento.
} 


\section{Notas}

Aprobación final del artículo: editora responsable Mag. Verónica Zorrilla de San Martín. Contribución de autoría: la elaboración del artículo es obra únicamente de la autora.

i Traducción libre. 